Open Access

\title{
Energy efficiency-based joint spectrum handoff and resource allocation algorithm for heterogeneous CRNs
}

\author{
Rong Chai ${ }^{*}{ }^{\dagger}$, Qin Hu, Qianbin Chen ${ }^{\dagger}$ and Zhiming Guo
}

\begin{abstract}
Cognitive radio networks (CRNs) are expected to improve spectrum utilization significantly by allowing secondary users (SUs) to opportunistically access the licensed spectrum of primary users (PUs). In an integrated network consisting of multiple heterogeneous CRNs, SUs with multiple interfaces may have to conduct inter-system or intra-system spectrum handoff due to the arrival of PUs or performance degradation on serving spectrum. In this case, designing an optimal spectrum handoff scheme which offers quality of service (QoS) guarantee and performance enhancement of the SUs is of particular importance. On the other hand, resource allocation strategy on target channel also plays an important role in affecting the transmission performance of handoff SUs. In this paper, we jointly design spectrum handoff and resource allocation strategy for handoff SUs in heterogeneously integrated CRNs. To achieve joint resource management among various $C R N s$, we propose a joint radio resource management architecture, based on which the proposed spectrum handoff and resource allocation scheme can be conducted. Jointly considering the transmission performance of the handoff SUs, we formulate the total energy efficiency of the SUs and design an optimization problem which maximizes the energy efficiency subject to spectrum handoff, QoS, and power constraints of the SUs. An iterative algorithm is proposed to solve the formulated nonlinear fractional optimization problem. Within each iteration, the optimization problem is transformed equivalently into two subproblems, i.e., power allocation subproblem of each SU-spectrum pair and spectrum handoff subproblem for all the SUs. The two subproblems are solved, respectively, through applying Lagrange dual method and the Kuhn-Munkres (K-M) algorithm. Numerical results demonstrate the efficiency of the proposed algorithm.
\end{abstract}

Keywords: Cognitive radio networks, Spectrum handoff, Resource allocation, Energy efficiency, Kuhn-Munkres algorithm

\section{Introduction}

Envisaged as a revolutionary technology to improve spectrum utilization, cognitive radio networks (CRNs) [1] have received considerable attentions from both academia and industry in recent years. In CRNs, secondary users (SUs) are allowed to share the spectrum of primary users (PUs) in an opportunistic manner without affecting the communication performance of the PUs. Multiple CRNs with heterogeneous access technologies may coexist in certain geographical regions resulting in heterogeneously

\footnotetext{
${ }^{*}$ Correspondence: chairong@cqupt.edu.cn

${ }^{\dagger}$ Equal contributors

Key Lab of Mobile Communication Technology, Chongqing University of Posts and Telecommunications, Chongwen Road, Chongqing, People's Republic of China
}

integrated CRNs, in which, SUs with multiple interfaces may access various CRNs.

Due to the arrival of PUs or performance degradation on serving channel, SUs may have to interrupt their current transmission and conduct spectrum handoff, i.e., switching to another spectrum belonging to the same or different CRNs. For an interrupted SU, the design of optimal spectrum handoff scheme is of particular importance for it may affect user quality of service (QoS) significantly. In addition, resource allocation strategy, for instance, the transmit power of SUs on handoff target channel also plays an important role in affecting the transmission performance of handoff SUs. While most of previous research works study spectrum handoff problem 
or resource allocation problem independently, these two problems are indeed closely related especially under the scenario that multiple SUs may conduct spectrum handoff simultaneously.

In this paper, we jointly consider spectrum handoff and resource allocation problem for handoff SUs in heterogeneously integrated CRNs. To achieve joint resource management among various CRNs, we propose a centralized joint radio resource management architecture. Jointly considering the transmission performance of the handoff SUs, we formulate the total energy efficiency of the SUs and design an optimization problem which maximizes the energy efficiency subject to spectrum handoff, QoS, and power constraints of the SUs. An iterative algorithm is proposed to solve the formulated nonlinear fractional optimization problem.

The major contributions of this paper are summarized as follows:

- We design a joint resource management architecture based on which the proposed joint spectrum handoff and resource allocation scheme can be conducted.

- Spectrum handoff scheme has been designed for single SU [2-5]. In this paper, we study the spectrum handoff problem of both single SU and multiple SUs. In addition, instead of merely stressing the service time and waiting delay of handoff SUs, we jointly consider the waiting delay, switching time, and the transmission performance of the handoff SUs on the target spectrum and propose an efficient joint spectrum handoff and resource allocation scheme for the handoff SUs.

- Spectrum handoff problem or resource allocation problem of SUs in CRNs has been studied independently in [2-16]. In this paper, we jointly consider spectrum handoff and resource allocation problem of SUs in heterogeneous CRNs and design a joint optimal strategy for all the SUs in terms of handoff target channel selection and transmit power allocation.

- To characterize the joint performance of all the handoff SUs, we stress the trade-off between transmission data rate and power consumption, examine the total energy efficiency of the SUs, and then formulate the joint spectrum handoff and resource allocation problem in heterogeneous CRNs as an energy efficiency maximization problem. Since the optimization problem formulated is a nonlinear fractional programming problem, which cannot be solved conveniently, we transform it to an equivalent optimization problem which can be solved based on iterative algorithm. Within each iteration, the optimization problem is transformed equivalently into two subproblems, i.e., power allocation subproblem of each SU-spectrum pair and spectrum handoff subproblem for all the
SUs. The two subproblems are then solved, respectively, through applying Lagrange dual method and the Kuhn-Munkres (K-M) algorithm.

The rest of the paper is organized as follows. Section 2 presents an overview of related works. Section 3 describes system model and the proposed joint resource management architecture. In Section 4, a channel queuing model is proposed and the interruption time of the handoff SUs is examined. A candidate spectrum selection scheme is proposed in Section 5. A joint spectrum handoff and resource allocation scheme is proposed for single handoff $\mathrm{SU}$ in Section 6. In Section 7, we propose a joint spectrum handoff and resource allocation scheme for multiple handoff SUs. In Section 8, the optimization problem formulated in Section 7 is solved. Simulation results are presented in Section 9. Finally, we conclude this paper in Section 10.

\section{Related works}

In this section, we present an overview of related works, including spectrum handoff schemes and resource allocation schemes of CRNs.

\subsection{Spectrum handoff schemes of CRNs}

In recent years, various spectrum handoff schemes have been designed for SUs in CRNs. In [2], the authors propose a probabilistic approach to determining the initial and target channel for a handoff SU in a CRN. The average sojourn time and the extended service time of the $\mathrm{SU}$ are analyzed, and the optimal spectrum handoff strategy is obtained through solving the optimization problem of minimizing both time metrics. In [3], a contention-aware channel selection algorithm is proposed, in which the secondary network detects the available licensed channels of SUs by exploiting cooperative spectrum sensing scheme, estimates the probability of channel collision, and then selects a less competitive channel for the SUs. In [4], a review of periodic sensing and handoff schemes is presented and the trade-off between the energy efficiency and the transmission reliability of SUs is examined. A switch-stay model is then proposed to achieve the tradeoff in light of sensing accuracy, the probability of collision, throughput, and delay constraints. In [5], the authors study spectrum handoff problem for multimedia applications in CRNs and propose a learning-based quality of experience (QoE)-driven spectrum handoff scheme which optimizes the quality of video transmissions in terms of handoff delay. The authors in [6] consider the stochastic characteristics of spatial and temporal spectrum availabilities in CRNs and develop a spectrum handoff scheme, which selects the handoff channel offering the maximum capacity. 
Previous research studies [2-6] mainly focus on designing spectrum handoff scheme for single SU in CRNs; however, it is highly possible that multiple SUs may have to perform spectrum handoff at the same time. For instance, multiple SUs occupying the licensed channel of one PU may have to interrupt their transmission and switch to other channels due to the arrival of the PU. The spectrum handoff problem of multiple SUs has been studied in $[7,8]$. The authors in [7] assume that the spectrum handoff of SUs should meet the constraint of handoff agility, i.e., SUs are only allowed to switch to their neighboring channels, and then formulate a continuous-time Markov model to analyze the forced termination and blocking probabilities of SUs. In [8], an analytical framework is presented to examine the impact of common control interface $(\mathrm{CCI})$ on the performance of spectrum handoff. The potential benefits and disadvantages of employing CCI for spectrum handoff are analyzed in terms of handoff delay, energy consumption, and spectrum utilization. Although $[7,8]$ stress the problem of spectrum handoff of multiple SUs, they mainly study the performance evaluation of handoff scheme instead of optimal handoff scheme design.

\subsection{Resource allocation schemes of CRNs}

To achieve transmission performance optimization of handoff SUs, resource allocation problems, in particular, transmit power allocation issue should be considered. Indeed, resource allocation problems in CRNs have been studied extensively in recent years. In [9], a multiuser orthogonal frequency-division multiple access (OFDMA) cognitive heterogeneous network (HetNet) is considered and the potential benefits of employing reconfigurable antennas (RA) are studied. The authors propose a game theoretical framework to jointly select the subcarriers as well as the RA state at each SU so that the overall capacity of the network can be maximized. The authors in [10] consider a CRN with imperfect spectrum sensing performance and propose an energy-aware centralized resource allocation scheme which takes into account relay selection, channel access, and power allocation jointly. The CRN is formulated as a partially observable Markov decision process (POMDP), based on which, an optimal joint resource allocation policy is derived. In [11], the authors investigate joint uplink subchannel and power allocation problem in cognitive small cells. Jointly considering the effects of cross-tier interference and imperfect channel state information (CSI), and the requirements on outage probability and transmission fairness, a cooperative Nash bargaining game model is formulated and a unified analytical framework is proposed to obtain the near optimal resource allocation strategy. The authors in [12] study joint power and channel allocation problem in CRNs offering heterogeneous services and propose an optimal resource allocation scheme which maximizes the capacity of all the SUs.

To meet the requirement on increasingly higher transmission rate, high transmit power is required, resulting in higher energy consumption at both user devices and base stations (BSs), which is highly undesired especially for energy-sensitive devices. To reduce power consumption and achieve high-energy efficiency of CRNs, energy-efficient resource allocation schemes have recently received great attentions [13-15]. Jiang et al. [13] presents a brief overview of energy-efficient schemes proposed for noncooperative CRNs, including spectrum sensing schemes, spectrum sharing schemes, and network deployment approaches. In [14], the authors study energyefficient opportunistic spectrum access strategies for an orthogonal frequency division multiplexing (OFDM)based CRN with multiple SUs. The worst energy efficiency and the average energy efficiency are, respectively, formulated and optimized for different application scenarios. In [15], approaches proposed for energy-efficient spectrum sensing and spectrum handoff are investigated and the trade-off between energy consumption and data transmission throughput is studied. In [16], an M/G/1 queuing model with preemptive resume priority is formulated for CRNs, the average waiting delay of SUs is analyzed, and the channel with the minimum service time is selected as the handoff channel.

Most of the previous works focus on either spectrum handoff scheme design or resource allocation scheme design for SUs in CRNs; indeed, it can be observed that resource allocation strategy on handoff target channel may affect the transmission performance of handoff SUs significantly; hence, it is highly desired to jointly design spectrum handoff and resource allocation strategy for SUs in CRNs. Indeed, in [6,13-16], the authors also consider resource management policy when designing spectrum handoff schemes for SUs; however, instead of designing the optimal transmit power strategy, they simply assume that the SUs transmit with the maximum transmit power or a fixed power on handoff target channels, thus may result in undesired transmission performance, especially in terms of energy efficiency. In this paper, we jointly study the spectrum handoff and resource allocation problem in heterogeneously integrated CRNs and propose an energy efficiency-based joint spectrum handoff and resource allocation scheme for handoff SUs.

\section{System model and proposed joint resource management architecture}

\subsection{System model}

In this paper, we consider an integrated network scenario consisting of one PN, multiple heterogeneous CRNs, and a number of PUs and SUs. As the licensed users of the 
PN, PUs are allowed to transmit to primary base stations (PBSs) through licensed channels. We assume that different channels of the PN are allocated to various PUs; hence, no co-channel interference exists among PUs. To support spectrum sharing between PUs and SUs, the channels of PUs are further divided into multiple subchannels, and each subchannel can be allocated to one SU for information interaction with the cognitive base stations (CBS) of one CRN. We assume that the SUs are equipped with multiple interfaces and are capable of accessing various CRNs but can only access one subchannel of a CRN at a given time interval.

We denote the number of PUs and SUs as $K$ and $M$, respectively, the total number of subchannels as $N$, and the $n$th subchannel as $C_{n}, 1 \leq n \leq N$. For convenience, we assume that the $k$ th channel is allocated to the $k$ th PU, $1 \leq k \leq K$. In Fig. 1, we plot the channel partition model considered in this paper.

\subsection{Proposed joint resource management architecture}

In the integrated networks of PN and CRNs, available radio resources need to be allocated in a coordinated way to guarantee QoS requirements of both PUs and SUs and to achieve performance enhancement of the networks. To achieve joint resource management of the networks in the heterogeneously integrated network scenario, a joint radio resource management (JRRM) architecture is proposed in this paper.

Figure 2 shows the proposed JRRM architecture, in which functional entities, including user RRMs (URRMs), local RRMs (LRRMs) and global RRM (GRRM), are introduced to tackle the dynamic information of the integrated networks and to conduct joint spectrum handoff and resource allocation for SUs. The major functions of URRMs, LRRMs, and GRRM are as follows.

URRM: functional module embedded in each PU and SU. URRMs collect and store CSI, device characteristics and service requirements, etc. Through interacting with the associated LRRMs, URRMs send their collected information to the network and receive spectrum handoff and resource allocation strategy.

LRRM: deployed in each PBS or CBS, being responsible for collecting and managing local resource status and resource management strategy through interacting with the associated URRMs and GRRM. More specifically, receiving network and service information from the associated URRMs and then forwarding to the GRRM, and receiving spectrum handoff and resource allocation strategy from GRRM and forwarding to URRMs.

GRRM: deployed over the PU and the CRNs. Through interacting with the associated LRRMs, the GRRM receives network and user state information and conducts the proposed joint spectrum handoff and resource allocation algorithm to obtain the optimal strategy and send back to the LRRMs.

It should be mentioned that the information interaction between GRRM, LRRMs, and URRMs can be performed over a common control channel [8]. Efficient random access control schemes and user scheduling schemes should be designed to guarantee the performance of information interaction between various functionality entities. In this paper, we assume that efficient information interaction between GRRM, LRRMs, and URRMs can be achieved and design joint spectrum handoff and resource allocation scheme based on the obtained information.

\section{Channel queuing model and interruption time analysis}

\subsection{Channel queuing model}

As PUs are of higher priority over SUs to access the licensed channels of PUs, once PUs reclaim their allocated channels, the SUs occupying the subchannels belonging to the PUs must interrupt their transmission. Without loss of generality, we assume that the service of SUs can tolerate certain interrupting delay; hence, when experiencing interruption, an SU may choose to stay at current subchannel or switch to other subchannel provided that the subchannel meets its interruption delay constraint. In the case that multiple SUs arrive at one subchannel, SUs may wait at the subchannel according to certain rules.

In this paper, an $M / G / 1$ queuing model is applied to characterize the spectrum-sharing behaviors between PUs and SUs [16]. To describe various priorities of PUs and SUs, the queuing model is partitioned into two sub-queues, i.e., high-priority sub-queues for PUs and low-priority sub-queues for SUs. The key features of the proposed M/G/1 queuing model are described as follows.

- PUs and SUs may arrive at various channels/subchannels with different rates. In the case that the channels are busy, users will wait in their

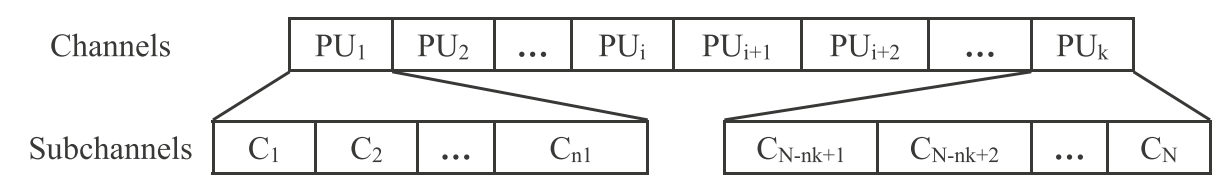

Fig. 1 Channel partition model. The channel and subchannel models of the PUs and the SUs 


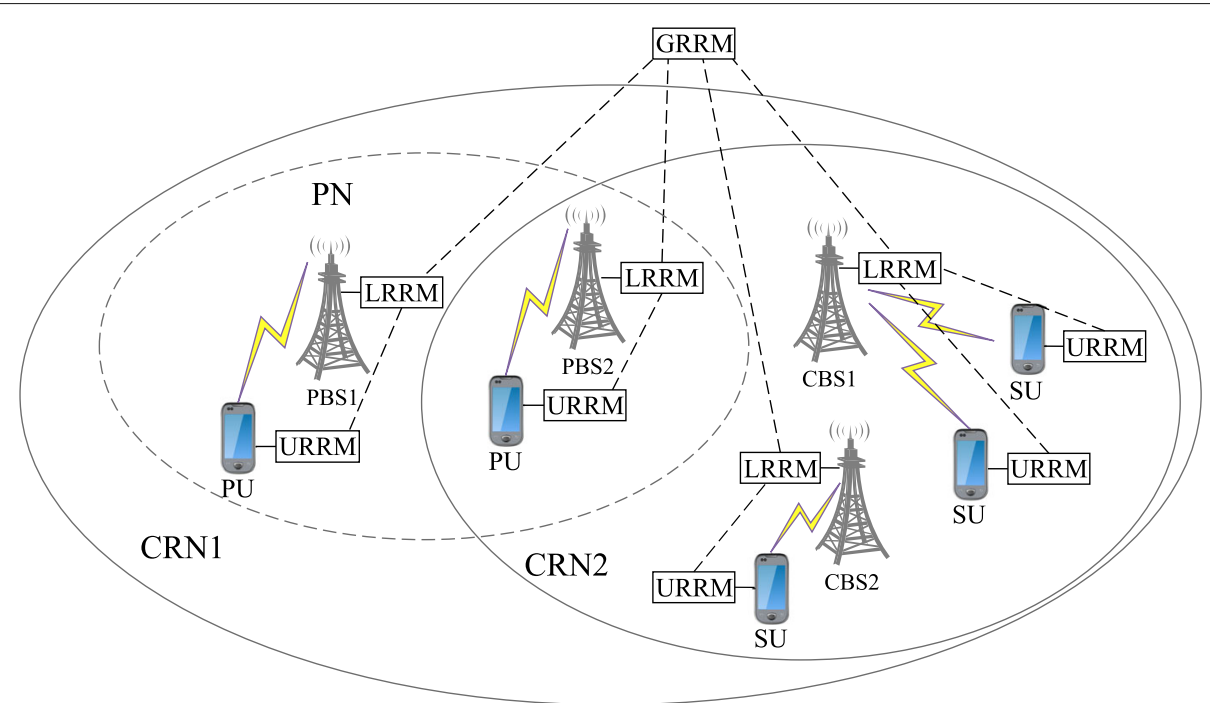

Fig. 2 Proposed joint resource management architecture. The proposed joint resource management architecture based on which the joint resource management scheme can be conducted

corresponding sub-queues, i.e., PUs wait in the highpriority sub-queues and SUs wait in the low-priority sub-queues till the channels/subchannels become available.

- Users waiting in the same sub-queue are allowed to access the channels/subchannels on the basis of first come first served (FCFS) scheduling scheme.

- To reduce the possibility of call drop, higher priority is given to interrupted SUs over new SUs, i.e., SUs with initial service requirement; thus, interrupted SUs will be put before new SUs in the low-priority sub-queues.

\subsection{Interruption time analysis}

In the case that one PU reclaims its allocated channel, the SUs occupying the subchannels belonging to the channel should interrupt their transmission, stay at current subchannel, or switch to another subchannel. For both cases, the interrupted SUs cannot resume their data communications until the PU or previously interrupted SUs have completed their transmission, thus resulting in waiting delay. If the interrupted SUs choose to switch to another subchannel, additional switch delay may occur due to spectrum handoff procedure.

In this section, we formulate the total interruption time of interrupted SUs as the sum of waiting delay and switching delay. Assuming that the $m$ th interrupted SU with the subchannel $n_{m}$ chooses the $n$th subchannel as its target subchannel, the corresponding interruption time can be calculated as:

$$
T_{m, n_{m}, n}^{(\mathrm{I})}=T_{m, n_{m}, n}^{(\mathrm{w})}+\delta_{n_{m}, n} T_{m, n_{m}, n}^{(\mathrm{s})}
$$

where $T_{m, n_{m}, n}^{(\mathrm{I})}, T_{m, n_{m}, n}^{(\mathrm{w})}$ and $T_{m, n_{m}, n}^{(\mathrm{s})}$ denote the interruption time, waiting delay, and switching delay of the $m$ th interrupted SU, respectively, and $\delta_{n_{m}, n}$ denotes the binary spectrum handoff index, i.e.,

$$
\delta_{n_{m}, n}=\left\{\begin{array}{l}
0, \text { if } n_{m}=n \\
1, \text { if } n_{m} \neq n .
\end{array}\right.
$$

In the following subsections, the waiting delay and the switching delay of interrupted SUs will be calculated, respectively.

\subsubsection{Waiting delay of interrupted SUs}

In this subsection, the waiting delay of interrupted SUs is analyzed for both staying case and switching case.

Staying case: In the case that an interrupted SU chooses to stay on its current subchannel, it will be put at the head of the sub-queue of the SUs and wait until the PU in the channel has completed its transmissions. Hence, the waiting delay can be expressed as the channel busy time due to the transmission of the PUs. Assuming the $n$th subchannel is originally allocated to the $k$ th PU, the waiting delay of the $m$ th interrupted SU can be calculated as:

$$
T_{m, n_{m}, n}^{(\mathrm{w})}=\mathrm{E}\left[T_{k}^{(\mathrm{p})}\right]
$$

where $T_{k}^{(\mathrm{p})}$ denotes the busy time of the $k$ th channel due to the transmission of the $k$ th $\mathrm{PU}$ and $\mathrm{E}[z]$ denotes the expectation value of $z$. E $\left[T_{k}^{(\mathrm{p})}\right]$ can be derived as follows.

Denoting $I_{k}^{(\mathrm{p})}$ as the idle period of the $k$ th channel, we can obtain:

$$
\mathrm{E}\left[I_{k}^{(\mathrm{p})}\right]=\frac{1}{\lambda_{k}^{(\mathrm{p})}}
$$


where $\lambda_{k}^{(\mathrm{p})}$ denotes the packet arrival rate of the PU on the $k$ th channel, $1 \leq k \leq K$. The utilization factor of the $k$ th channel, denoted by $\rho_{k}^{(\mathrm{p})}$, can be expressed as:

$$
\rho_{k}^{(\mathrm{p})}=\frac{\mathrm{E}\left[T_{k}^{(\mathrm{p})}\right]}{\mathrm{E}\left[T_{k}^{(\mathrm{p})}\right]+\mathrm{E}\left[I_{k}^{(\mathrm{p})}\right]}=\frac{\lambda_{k}^{(\mathrm{p})}}{\mu_{k}^{(\mathrm{p})}}
$$

where $\mu_{k}^{(\mathrm{p})}$ denotes the service rate of the PU on the $k$ th channel, $1 \leq k \leq K$. Combining (4) and (5), we obtain:

$$
\mathrm{E}\left[T_{k}^{(\mathrm{p})}\right]=\frac{1}{\mu_{k}^{(\mathrm{p})}-\lambda_{k}^{(\mathrm{p})}} .
$$

Switching case: In the case that an interrupted SU chooses to switch to another subchannel, it will be put into the low-priority sub-queue of the target subchannel and has to wait for the PU or the SU transmitting on the subchannel to complete its transmission. Furthermore, the interrupted SU also needs to wait for the PUs and the previously interrupted SUs waiting in the queue to complete their transmission. Hence, the waiting delay of the interrupted SU can be calculated as the sum of the busy time of the channel due to the transmission of the PU or the SU and the remaining service time of the subchannel [16]. Denoting $T_{m, n_{m}, n}^{(\mathrm{b})}$ as the busy time of the subchannel and $T_{m, n_{m}, n}^{(\mathrm{r})}$ as the remaining service time of the subchannel, we can obtain:

$$
T_{m, n_{m}, n}^{(\mathrm{w})}=T_{m, n_{m}, n}^{(\mathrm{b})}+T_{m, n_{m}, n}^{(\mathrm{r})}
$$

where $T_{m, n_{m}, n}^{(\mathrm{b})}$ can be calculated as:

$$
T_{m, n_{m}, n}^{(\mathrm{b})}=\frac{1}{2} \mathrm{E}\left[T_{k}^{(\mathrm{p})}\right]+\frac{1}{2} \mathrm{E}\left[T_{n}^{(\mathrm{s})}\right]
$$

where $T_{n}^{(\mathrm{s})}$ denotes the transmission time of one SU on the $n$th subchannel and can be calculated as:

$$
\mathrm{E}\left[T_{n}^{(\mathrm{s})}\right]=\frac{1}{\mu_{n}^{(\mathrm{s})}-\lambda_{n}^{(\mathrm{s})}}
$$

where $\lambda_{n}^{(\mathrm{s})}$ and $\mu_{n}^{(\mathrm{s})}$ denote the packet arrival rate and service rate, respectively, of the SU accessing the $n$th subchannel, $1 \leq n \leq N$. The remaining service time of the $n$th subchannel can be calculated as:

$$
T_{m, n_{m}, n}^{(\mathrm{r})}=\frac{\lambda_{k}^{(\mathrm{p})}}{\left(\mu_{k}^{(\mathrm{p})}\right)^{2}}+\frac{\lambda_{n}^{(\mathrm{s})}}{\left(\mu_{n}^{(\mathrm{s})}\right)^{2}} .
$$

Combining (7)-(10), we obtain:

$$
\begin{aligned}
T_{m, n_{m}, n}^{(\mathrm{w})}= & \frac{1}{2\left(\mu_{k}^{(\mathrm{p})}-\lambda_{k}^{(\mathrm{p})}\right)}+\frac{1}{2\left(\mu_{n}^{(\mathrm{s})}-\lambda_{n}^{(\mathrm{s})}\right)}+\frac{\lambda_{k}^{(\mathrm{p})}}{\left(\mu_{k}^{(\mathrm{p})}\right)^{2}} \\
& +\frac{\lambda_{n}^{(\mathrm{s})}}{\left(\mu_{n}^{(\mathrm{s})}\right)^{2}} .
\end{aligned}
$$

\subsubsection{Switching delay of interrupted SUs}

In the case that an interrupted SU decides to switch from the $n_{m}$ th subchannel to the $n$th subchannel, intra-system or inter-system handoff may occur as the $n_{m}$ th subchannel and the $n$th subchannel may belong to the same CRN or different CRNs. In addition, as one SU may perform spectrum switch and radio frequency (RF) reconfiguration before or after a PU reoccupies the channel, the corresponding spectrum handoff is referred to as proactive handoff and reactive handoff, respectively. Jointly considering these handoff scenarios, we obtain four types of spectrum handoff, i.e., proactive intra-system handoff, proactive inter-system handoff, reactive intra-system handoff, and reactive inter-System handoff; the corresponding switching delay is examined as follows.

Proactive intra-system handoff Denoting $T^{(\mathrm{s}, 1)}$ as the switching delay of proactive intra-system handoff, we obtain:

$$
T^{(\mathrm{s}, 1)}=t_{\mathrm{syn}}^{\mathrm{sen}}+t_{\mathrm{sen}}+t_{\mathrm{dec}}+t_{\mathrm{switch}}+t_{\mathrm{syn}}^{\mathrm{tx}}
$$

where $t_{\text {syn }}^{\text {sen }}$ denotes the synchronization time for spectrum sensing, $t_{\text {sen }}$ denotes the time period for sensing spectrum resource, $t_{\mathrm{dec}}$ denotes the time period for determining a target handoff spectrum, $t_{\text {switch }}$ denotes the time period required for switching from current subchannel to target subchannel, and $t_{\mathrm{syn}}^{\mathrm{tx}}$ denotes the time duration required for conducting synchronization with target subchannel.

Proactive inter-system handoff In the case that a proactive handoff is performed between two heterogeneous CRNs, the reconfiguration of RF front end is required. Denoting the time duration required for reconfiguration

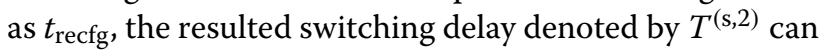
be expressed as:

$$
T^{(\mathrm{s}, 2)}=t_{\mathrm{recfg}}+T^{(\mathrm{s}, 1)} .
$$

Reactive intra-system handoff In the case that a reactive handoff is performed inside one CRN, a handoff preparation time, denoted by $t_{\mathrm{prep}}$, is required to collect measurement information and determine handoff target subchannel; thus, the corresponding switching delay denoted by $T^{(\mathrm{s}, 3)}$ can be expressed as:

$$
T^{(\mathrm{s}, 3)}=t_{\text {prep }}+T^{(\mathrm{s}, 1)} \text {. }
$$

Reactive inter-system handoff In the case that a reactive handoff is performed between two heterogeneous CRNs, handoff preparation and reconfiguration of RF front end are both required. As a result, the switching delay denoted by $T^{(\mathrm{s}, 4)}$ can be expressed as:

$$
T^{(\mathrm{s}, 4)}=t_{\mathrm{recfg}}+T^{(\mathrm{s}, 3)} \text {. }
$$




\section{Candidate handoff spectrum selection scheme}

Interrupted SUs with various user services may pose different QoS requirements on transmission subchannels. In this paper, we assume interrupted SUs may have different interruption delay and data rate requirements, which place constraints on handoff target subchannel. To reduce the computation complexity of the proposed spectrum handoff and resource allocation scheme, we present a candidate spectrum selection scheme which selects the qualified spectrum among all the available spectrum based on the QoS requirements of interrupted SUs; then, the proposed joint spectrum handoff and resource allocation scheme only applies to the candidate spectrum of the SUs.

In the following subsections, we first examine both interruption delay constraint and data rate constraint, based on which, we discuss the formation of candidate handoff spectrum set.

\subsection{Interruption delay constraint}

The transmission interruption of SUs resulted from waiting in subchannel queues or performing spectrum handoff might be unacceptable for delay sensitive services. Hence, for a particular interrupted SU, the handoff target subchannel should meet certain interruption delay constraint. Denoting $T_{m}^{\max }$ as the maximum acceptable interruption delay of the $m$ th interrupted SU, the $n$th subchannel can be selected as the handoff target subchannel of the SU only if it meets the following delay constraint:

$$
T_{m, n_{m}, n}^{(\mathrm{I})} \leq T_{m}^{\max }
$$

\subsection{Data rate constraint}

To offer data transmission service to an interrupted SU with a minimum data rate constraint, a handoff target subchannel has to meet the data rate requirement of the SU. Denoting $R_{m, n}$ as the achievable data rate of the $m$ th interrupted SU on the $n$th subchannel, according to Shannon formula [17], we can obtain:

$$
R_{m, n}=B_{n} \log _{2}\left(1+\frac{P_{m, n} h_{m, n}^{2}}{\sigma^{2}}\right)
$$

where $B_{n}$ denotes the bandwidth of the $n$th subchannel, $P_{m, n}$ denotes the transmit power of the $m$ th interrupted SU on the $n$th subchannel, and $\sigma^{2}$ denotes the noise power of the link from the $m$ th interrupted SU to its CBS on the $n$th subchannel. Without loss of generality, the noise power is assumed to be a constant for all the links of the CRNs in this paper, $h_{m, n}$ denotes the subchannel gain of the link from the $m$ th interrupted SU to its CBS on the $n$th subchannel. In this paper, we assume the channel model follows Friis free-space propagation model [18], and the subchannel gain $h_{m, n}$ can be expressed as:

$$
h_{m, n}=\left(\frac{c}{4 \pi f_{n} d_{m}}\right)^{2}
$$

where $c$ denotes the speed of light, $f_{n}$ denotes carrier frequency of the $n$th subchannel, and $d_{m}$ denotes the distance from the $m$ th SU to its corresponding CBS. Denoting $R_{m}^{\min }$ as the minimum data rate requirement of the $m$ th interrupted SU, the data rate constraint can be expressed as:

$$
R_{m, n} \geq R_{m}^{\min }
$$

As $P_{m, n}$ in (17) is one of the optimization variables in our proposed spectrum handoff and resource allocation scheme, the exact $R_{m, n}$ cannot be obtained before completing the optimization process and obtaining the optimal $P_{m, n}$. However, as each user device may have to meet a maximum transmit power requirement due to hardware and signal processing constraint, we can instead examine the maximum achievable data rate of the $m$ th SU on the $n$th subchannel through replacing $P_{m, n}$ by its maximum value. Denoting $P_{m}^{\max }$ as the maximum permissible transmit power of the $m$ th SU, the transmit power of the SU should be less than $P_{m}^{\max }$, i.e.,

$$
P_{m, n} \leq P_{m}^{\max }
$$

Combining (17), (19), and (20), we can rewrite the data rate constraint as:

$$
R_{m, n}^{\max }=B_{n} \log _{2}\left(1+\frac{P_{m}^{\max } h_{m, n}^{2}}{\sigma^{2}}\right) \geq R_{m}^{\min } .
$$

In the case that the candidate handoff spectrum set is empty, indicating that there is no subchannels meeting the transmission constraints of the interrupted SUs, thus, the SUs cannot conduct handoff successfully, resulting in transmission interruption.

\subsection{Candidate handoff spectrum set}

Jointly considering the interruption delay constraint and data rate constraint of SUs, we can obtain that the $n$th subchannel can be selected as the handoff target subchannel of the $m$ th SU of which the current subchannel is $n_{m}$, only if it meets the conditions specified in both (16) and (21). Denoting $\Phi$ as the set of all the subchannels and $\Phi_{m, n_{m}}$ as the set of candidate handoff subchannel of the $m$ th SU with the current subchannel being $n_{m}$, we obtain:

$$
\Phi_{m, n_{m}}=\left\{\mathrm{C}_{n} \mid T_{m, n_{m}, n}^{(\mathrm{I})} \leq T_{m}^{\max }, R_{m, n}^{\max } \geq R_{m}^{\min }, \mathrm{C}_{n} \in \Phi\right\} .
$$

Among all the candidate handoff subchannels of the $m$ th interrupted SU, i.e., $C_{n} \in \Phi_{m, n_{m}}$, the subchannel offering the optimal performance will be selected and the 
corresponding optimal transmit power of the SU will be designed, as discussed in the following two sections.

\section{Proposed joint optimization scheme: single SU case}

In this section, we consider the case that only one $\mathrm{SU}$ is interrupted and design an optimal joint spectrum handoff and power allocation scheme for the SU. While experiencing transmission interruption, the SU may tend to choose the subchannel corresponding to the optimal transmission performance. In this paper, to stress the importance of both the transmission rate and power consumption of the interrupted SUs, and to achieve the trade-off between the two metrics, the energy efficiency of the interrupted SUs on the target subchannel is examined and optimized in terms of the transmit power of the SUs.

For convenience, we denote the single interrupted SU as the first SU, i.e., we set $m=1$ in the formulas of the previous sections. The energy efficiency of the interrupted SU when transmitting on the $n$th subchannel denoted by $\eta_{n}$ can be defined as the ratio of the achievable data rate and the power consumption of the SU on the $n$th subchannel, i.e.,

$$
\eta_{n}=\frac{R_{1, n}}{P_{1, n}+P^{\mathrm{cir}}}
$$

where $P^{\text {cir }}$ denotes the circuit consumption power of the interrupted $\mathrm{SU}$, which is assumed to be a constant for all the SUs in this paper.

To achieve high-energy efficiency, the interrupted SU with current subchannel being $n_{1}$ may choose the $n$th subchannel which offers the maximum energy efficiency among all the candidate subchannels as the handoff target subchannel. However, it can be seen from (23) that the energy efficiency, i.e., $\eta_{n}$, varies with the transmit power $P_{1, n}$ for given channel and device characteristics; thus, it is difficult to examine and compare the energy efficiency of various subchannels. To stress this problem, we propose a two-step algorithm which consists of both power allocation and spectrum handoff subalgorithms. More specifically, we first conduct optimal power allocation subalgorithm on the $n$th subchannel, i.e., optimizing $\eta_{n}$ in terms of $P_{1, n}$ to obtain the maximum $\eta_{n}$, denoted by $\eta_{n}^{*}$, for $\mathrm{C}_{n} \in \Phi_{1, n_{1}}, 1 \leq n \leq N$, and then apply optimal spectrum handoff subalgorithm, i.e., choosing the optimal subchannel corresponding to the maximum $\eta_{n}^{*}$.

The detailed algorithm is discussed as follows. For the $n$th subchannel, $\mathrm{C}_{n} \in \Phi_{1, n_{1}}, 1 \leq n \leq N$, the optimal power allocation problem can be formulated as:

$$
\begin{array}{cc}
\max _{P_{1, n}} & \eta_{n} \\
\text { s.t. } & \mathrm{C} 1: R_{1, n} \geq R_{1}^{\min }, \\
& \mathrm{C} 2: P_{1, n} \leq P_{1}^{\max } .
\end{array}
$$

For a given range of $P_{1, n}$, i.e., $0<P_{1, n} \leq P_{1}^{\max }$, the optimal energy efficiency of the interrupted SU on the $n$th subchannel, denoted by $\eta_{n}^{*}$, can be obtained through solving above problem via numerical method or optimization techniques [19].

Given $\eta_{n}^{*}$, we can then conduct optimal spectrum handoff subalgorithm through which the $n^{*}$ th subchannel offering the maximum $\eta_{n}^{*}$ is selected as the optimal handoff subchannel among all $C_{n} \in \Phi_{1, n_{1}}, 1 \leq n \leq N$, i.e.,

$$
\mathrm{C}_{n^{*}}=\arg \max _{\mathrm{C}_{n} \in \Phi_{1, n_{1}}}\left(\eta_{n}^{*}\right)
$$

\section{Proposed joint optimization scheme: multiple SUs' case}

It can be seen from the previous section that in the case that the transmission of one SU is interrupted, the optimal spectrum handoff and power allocation problem can be solved through designing the optimal transmit power strategy and then selecting the optimal subchannel, which offers the maximal energy efficiency. In the case that multiple SUs may perform spectrum handoff simultaneously, allowing each interrupted SU to select its optimal subchannel may not be feasible for resource competition among handoff SUs exists. In this paper, we propose to jointly consider the performance of interrupted SUs and design an optimal spectrum handoff and power allocation strategy which achieves the performance optimization of all the SUs.

\subsection{Total energy efficiency of interrupted SUs}

In this paper, we formulate the joint performance of interrupted SUs as the total energy efficiency, which is defined as the ratio of the sum rate and total power consumption of the interrupted SUs [20, 21], i.e.,

$$
\eta^{\text {tot }}=\frac{R^{\text {tot }}}{P^{\text {tot }}}
$$

where $R^{\text {tot }}$ and $P^{\text {tot }}$ denote, respectively, the sum rate and the total power consumption of the interrupted SUs; $R^{\text {tot }}$ can be expressed as:

$$
R^{\mathrm{tot}}=\sum_{m=1}^{M} R_{m}
$$

where $R_{m}$ denotes the data rate of the $m$ th interrupted SU, which can be calculated as:

$$
R_{m}=\sum_{n=1}^{N} x_{m, n} R_{m, n}
$$

where $x_{m, n} \in\{0,1\}$ is a binary spectrum handoff variable, i.e., $x_{m, n}=1$, if the $m$ th SU selects the $n$th subchannel as its handoff target subchannel; otherwise, $x_{m, n}=0$. As it is assumed that each interrupted SU can only select one 
subchannel for handoff, and each subchannel can only be allocated to one interrupted $\mathrm{SU}$, we obtain:

$$
\begin{aligned}
& \sum_{n=1}^{N} x_{m, n} \leq 1, \\
& \sum_{m=1}^{M} x_{m, n} \leq 1 .
\end{aligned}
$$

The total power consumption of the interrupted SUs can be calculated as the sum of power consumption of all the SUs, i.e.,

$$
P^{\text {tot }}=\sum_{m=1}^{M} P_{m}
$$

where $P_{m}$ denotes the power consumption of the $m$ th interrupted $\mathrm{SU}$, which can be calculated as:

$$
P_{m}=\sum_{n=1}^{N} x_{m, n}\left(P_{m, n}+P^{\mathrm{cir}}\right) \text {. }
$$

\subsection{Optimization problem formulation}

In this paper, we propose to design a joint spectrum handoff and power allocation scheme which achieves the maximum energy efficiency of all the interrupted SUs. By applying optimization theory, the joint spectrum handoff and power allocation problem can be formulated as follows:

$$
\begin{array}{cl}
\max _{x_{m, n}, P_{m, n}} & \eta^{\text {tot }} \\
\text { s.t. } & \mathrm{C} 1: x_{m, n} \in\{0,1\}, 1 \leq m \leq M, 1 \leq n \leq N, \\
& \mathrm{C} 2: \sum_{n=1}^{N} x_{m, n} \leq 1,1 \leq m \leq M \\
& \mathrm{C} 3: \sum_{m=1}^{M} x_{m, n} \leq 1,1 \leq n \leq N \\
& \mathrm{C} 4: x_{m, n}=0, \text { if } \mathrm{C}_{n} \notin \Phi_{m, n_{m}}, 1 \leq m \leq M, \\
& 1 \leq n, n_{m} \leq N, \\
& \text { C5 }: R_{m} \geq R_{m}^{\min }, 1 \leq m \leq M, 1 \leq n \leq N, \\
& \text { C6 }: \sum_{n=1}^{N} x_{m, n} P_{m, n} \leq P_{m}^{\max }, 1 \leq m \leq M .
\end{array}
$$

\section{Solution of the optimization problem}

The optimization problem formulated in (33) is a nonconvex problem with the objective function being a nonlinear fractional function, which cannot be solved conveniently using traditional optimization tools. In this section, the optimization objective function is transformed into a convex function and the corresponding optimization problem is solved using iterative algorithm [22].

\subsection{Iterative algorithm-based energy efficiency maximization}

To transform the objective function defined in (33), we denote $q$ as the energy efficiency of the interrupted SUs, i.e.,

$$
q=\frac{R^{\mathrm{tot}}\left(x_{m, n}, P_{m, n}\right)}{P^{\operatorname{tot}}\left(x_{m, n}, P_{m, n}\right)}
$$

Denote $q^{*}$ as the optimal value of $q$, we obtain:

$$
q^{*}=\frac{R^{\mathrm{tot}}\left(x_{m, n}^{*}, P_{m, n}^{*}\right)}{P^{\operatorname{tot}}\left(x_{m, n}^{*}, P_{m, n}^{*}\right)}=\max _{x_{m, n}, P_{m, n}}\left(\frac{R^{\mathrm{tot}}\left(x_{m, n}, P_{m, n}\right)}{P^{\operatorname{tot}}\left(x_{m, n}, P_{m, n}\right)}\right)
$$

It can be proved that the maximum energy efficiency $q^{*}$ is achieved if and only if the following condition meets [20]:

$$
\begin{aligned}
& \max _{x_{m, n}, P_{m, n}}\left(R^{\mathrm{tot}}\left(x_{m, n}, P_{m, n}\right)-q^{*} P^{\mathrm{tot}}\left(x_{m, n}, P_{m, n}\right)\right) \\
& =R^{\mathrm{tot}}\left(x_{m, n}^{*}, P_{m, n}^{*}\right)-q^{*} P^{\mathrm{tot}}\left(x_{m, n}^{*}, P_{m, n}^{*}\right)=0 .
\end{aligned}
$$

Hence, the optimization problem formulated in (33) can be transformed into the following problem:

$$
\begin{array}{cl}
\max _{q, x_{m, n}, P_{m, n}} & R^{\mathrm{tot}}\left(x_{m, n}, P_{m, n}\right)-q P^{\mathrm{tot}}\left(x_{m, n}, P_{m, n}\right) \\
\text { s.t. } & \mathrm{C} 1-\mathrm{C} 6 \text { in }(33)
\end{array}
$$

Examining the above optimization problem, we can observe that for a given energy efficiency $q$, the problem is still a joint spectrum handoff and power allocation problem with the objective function being a convex function, thus can be solved (as we will discuss in the following subsections) to obtain the locally optimal spectrum handoff and power allocation strategy, based on which the energy efficiency $q$ can be updated. Repeating the process until the convergence condition meets, we can obtain the globally optimal energy efficiency and joint spectrum handoff and power allocation strategy.

The above iterative process can be implemented through an iterative algorithm, which proceeds as follows. Starting from an initial value of $q$, we can obtain the locally optimal $x_{m, n}$ and $P_{m, n}$ through solving the spectrum handoff and power allocation subproblem; then, $q$ is updated based on (34). For the updated $q$, the spectrum and power allocation subproblem can be resolved to obtain updated $x_{m, n}$ and $P_{m, n}$, the process continues until the convergence condition, i.e., $\left|R^{\text {tot }}\left(x_{m, n}, P_{m, n}\right)-q P^{\text {tot }}\left(x_{m, n}, P_{m, n}\right)\right| \leq \omega_{1}$ meets, where $\omega_{1}$ denotes the maximum tolerance. The proposed algorithm is summarized in Algorithm 1 and the convergence of the algorithm can be guaranteed [20].

\subsection{Locally optimal spectrum handoff and power allocation algorithm}

For a given $q$, the joint spectrum handoff and power allocation problem can be formulated as follows:

$$
\begin{gathered}
\max _{x_{m, n}, P_{m, n}} R^{\text {tot }}\left(x_{m, n}, P_{m, n}\right)-q P^{\text {tot }}\left(x_{m, n}, P_{m, n}\right) \\
\text { s.t. } \quad \text { C } 1-\text { C6 in }(33)
\end{gathered}
$$




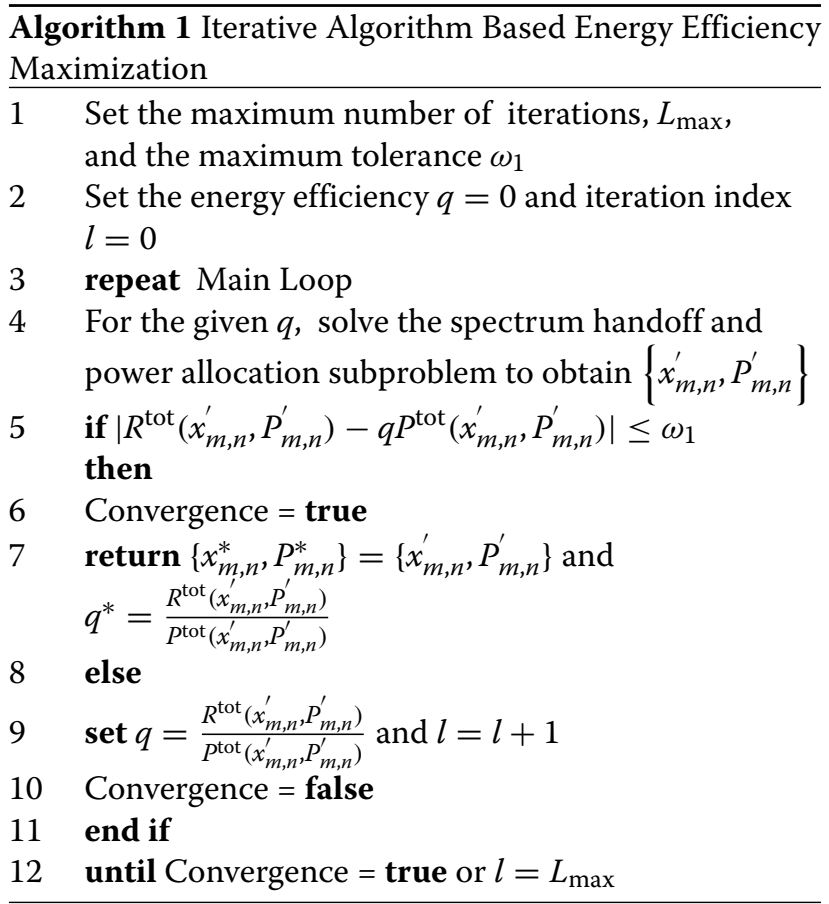

The objective function in (37) can be rewritten as:

$$
\sum_{m=1}^{M} \sum_{n=1}^{N} x_{m, n}\left(R_{m, n}-q\left(P_{m, n}+P^{\mathrm{cir}}\right)\right)
$$

It can be seen from (39) that if we assume the $m$ th interrupted SU selects the $n$th subchannel as its handoff target subchannel, i.e., $x_{m, n}=1$, for the given SU-spectrum pair, maximizing (39) is equivalent to maximizing $R_{m, n}-$ $q\left(P_{m, n}+P^{\text {cir }}\right)$ in terms of $P_{m, n}$, which can be referred to as power allocation subproblem. From the constraints C1-C3 in (33), we can obtain that the power allocation for various SU-spectrum pairs is independent, thus can be solved individually. Once we have obtained the optimal power allocation strategy for SU-spectrum pairs, we can then solve spectrum handoff subproblem to obtain the optimal $x_{m, n}$. Hence, the problem formulated in (37) can be transformed equivalently into two subproblems, i.e., power allocation subproblem of one SU-spectrum pair and the spectrum handoff subproblem of all the SUs. In the following subsections, we solve the two subproblems, respectively.

\subsubsection{Optimal power allocation subproblem}

We assume that the $m$ th interrupted SU chooses the $n$th subchannel as handoff target subchannel, i.e., $x_{m, n}=1$, $\mathrm{C}_{n} \in \Phi_{m, n_{m}}, 1 \leq m \leq M, 1 \leq n, n_{m} \leq N$, and the optimal power allocation subproblem of the SU-spectrum pair can be formulated as:

$$
\begin{array}{cc}
\max _{P_{m, n}} & R_{m, n}-q\left(P_{m, n}+P^{\mathrm{cir}}\right), \\
\text { s.t. } & \mathrm{C} 1: P_{m, n} \leq P_{m}^{\max } \\
& \mathrm{C} 2: R_{m, n} \geq R_{m}^{\min }
\end{array}
$$

It can be proved easily that the above optimization problem is a convex problem which can be solved using traditional optimization tools. In this paper, we apply Lagrange dual method to solve the problem. The Lagrangian function of (40) can be written as:

$$
\begin{aligned}
& L\left(\mu_{m}, \gamma_{m}, P_{m, n}\right)=R_{m, n}-q\left(P_{m, n}+P^{\mathrm{cir}}\right) \\
& -\mu_{m}\left(P_{m, n}-P_{m}^{\max }\right)-\gamma_{m}\left(R_{m}^{\min }-R_{m, n}\right)
\end{aligned}
$$

where $\mu_{m}, \gamma_{m} \geq 0$ are the Lagrange multipliers corresponding to the maximum power constraint and the minimum data rate constraint, respectively. The Lagrange dual problem of (40) can be formulated as follows:

$$
\begin{gathered}
\min _{\mu_{m}, \gamma_{m}} \max _{P_{m, n}} L\left(\mu_{m}, \gamma_{m}, P_{m, n}\right) \\
\text { s.t. } \quad \mu_{m} \geq 0, \gamma_{m} \geq 0 .
\end{gathered}
$$

The above dual problem can be solved by optimizing the transmit power for a fixed set of Lagrange multipliers and updating the Lagrange multipliers iteratively. For a given set of Lagrange multipliers $\left\{\mu_{m}, \gamma_{m}\right\}$, we can obtain the locally optimal transmit power through calculating the derivative of $L\left(\mu_{m}, \gamma_{m}, P_{m, n}\right)$ over $P_{m, n}$ and setting it to 0 , i.e.,

$$
\frac{\partial L\left(\mu_{m}, \gamma_{m}, P_{m, n}\right)}{\partial P_{m, n}}=0 .
$$

Solving the equation over $P_{m, n}$, we obtain:

$$
P_{m, n}=\left[\frac{\left(1+\gamma_{m}\right) B_{n}}{\left(\mu_{m}+q\right) \ln 2}-\frac{\sigma^{2}}{h_{m, n}^{2}}\right]^{+}
$$

where $[z]^{+}=\max (0, z)$. The Lagrange multipliers $\mu_{m}$ and $\gamma_{m}$ in (44) can be updated based on the gradient method, i.e.,

$$
\begin{aligned}
& \mu_{m}(t+1)=\left[\mu_{m}(t)-\varepsilon_{1}\left(P_{m}^{\max }-P_{m, n}\right)\right]^{+}, \\
& \gamma_{m}(t+1)=\left[\gamma_{m}(t)-\varepsilon_{2}\left(R_{m, n}-R_{m}^{\min }\right)\right]^{+}
\end{aligned}
$$

where $\varepsilon_{1}$ and $\varepsilon_{2}$ are stepsize. The proposed Lagrange dual method-based power allocation algorithm is shown in Algorithm 2.

\subsubsection{Spectrum handoff subproblem}

Through assuming $x_{m, n}=1, \mathrm{C}_{n} \in \Phi_{m, n_{m}}, 1 \leq m \leq M$, $1 \leq n, n_{m} \leq N$, we can obtain the locally optimal power allocation strategy, denoted as $P_{m, n}^{*}$. Substituting $P_{m, n}$ by $P_{m, n}^{*}$ in (39), we obtain: 


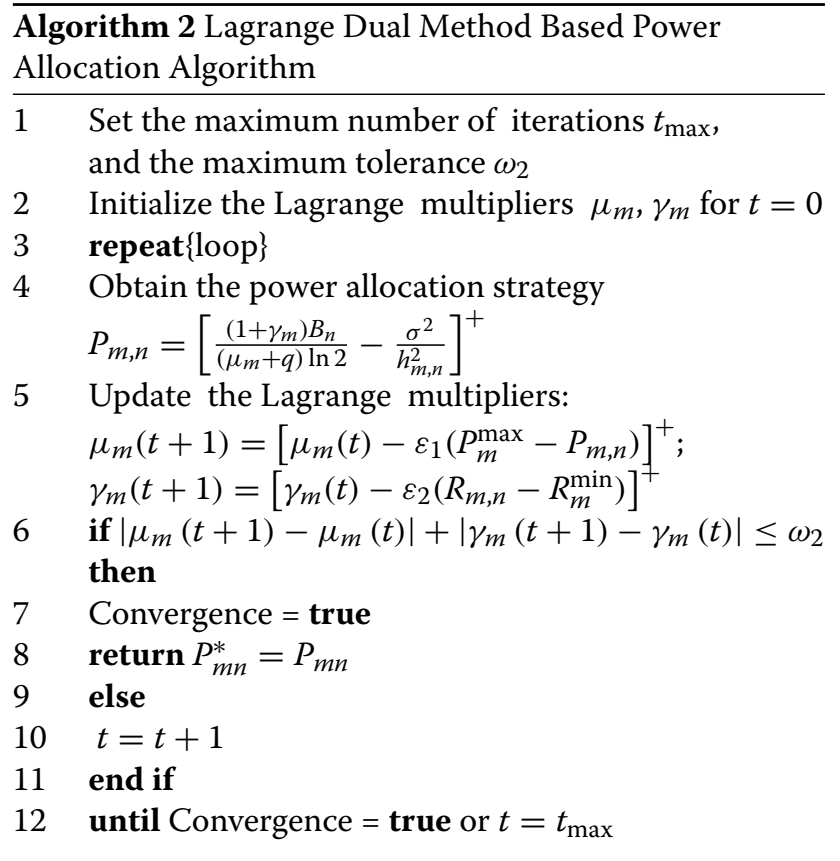

$\sum_{m=1}^{M} \sum_{n=1}^{N} x_{m, n}\left(R_{m, n}^{*}-q\left(P_{m, n}^{*}+P^{\mathrm{cir}}\right)\right)$

where $R_{m, n}^{*}=B_{n} \log _{2}\left(1+\frac{P_{m, n}^{*} h_{m, n}^{2}}{\sigma^{2}}\right)$. For a given $P_{m, n}^{*}$, $R_{m, n}^{*}-q\left(P_{m, n}^{*}+P^{\mathrm{cir}}\right)$ is a constant; therefore, the problem of maximizing (47) is simplified as selecting the optimal $x_{m, n}$, which can be solved based on the following optimal spectrum handoff subproblem:

$$
\begin{array}{ll}
\max _{x_{m, n}} & \sum_{m=1}^{M} \sum_{n=1}^{N} x_{m, n}\left(R_{m, n}^{*}-q\left(P_{m, n}^{*}+P^{\mathrm{cir}}\right)\right) \\
\text { s.t. } & \mathrm{C} 1-\mathrm{C} 4 \text { in }(33) .
\end{array}
$$

The optimization problem formulated in (48) is a linear binary optimization problem which is equivalent to a bipartite matching problem in graph theory and can be solved based on the K-M algorithm [23]. Before applying the K-M algorithm, we first introduce some related concepts and theorem as follows.

Complete bipartite graph: Given a graph $G=(V ; E)$ which consists of a set $V$ of vertices and a set $E$ of edges which connect a pairs of vertices, if the set $V$ is divided into two disjoint and nonempty sets, $X$ and $Y$, i.e., $V=X \cup Y$, every edge in $E$ joins one vertex in $X$ to another vertex in $Y$, and no edge connects two vertices of the same set, we call $G$ a complete bipartite graph.
Complete bipartite graph: Given a graph $G=(V ; E)$ which consists of a set $V$ of vertices and a set $E$ of edges which connect a pairs of vertices, if the set $V$ is divided into two disjoint and nonempty sets, $X$ and $Y$, i.e., $V=X \cup Y$, every edge in $E$ joins one vertex in $X$ to another vertex in $Y$, and no edge connects two vertices of the same set, we call $G$ a complete bipartite graph.

Weighted complete bipartite graph: A complete bipartite graph $G=(V ; E)$ is a weighted complete bipartite graph if any edge $e_{x, y} \in E$ connecting $x \in X$ and $y \in Y$ is assigned a nonnegative weight $w(x, y)$.

Maximum matching: A matching $H$ of graph $G=(V ; E)$ is defined as a subset $H \subseteq E$ which meets the condition that for $\forall e_{x, y}, e_{x^{\prime}, y^{\prime}} \in H, e_{x, y}, e_{x^{\prime}, y^{\prime}}$ are not adjacent in $G$. The size of a matching $H$, denoted by $|H|$, is defined as the number of edges contained in $H$. A matching $H$ is called a maximum matching if the condition $\left|H^{\prime}\right| \leq|H|$ holds, where $H^{\prime}$ denotes any other matchings of $G$.

Optimal matching: The maximum matching $H$ of graph $G=(V ; E)$ is called an optimal matching if it achieves the maximum sum weight, i.e.,

$$
\sum_{e_{x, y} \in H} w(x, y) \geq \sum_{e_{x, y} \in H^{\prime}} w(x, y)
$$

Feasible vector labeling: A real valued function $l$ such that for any $x \in X$ and $y \in Y, l(x)+l(y) \leq w(x, y)$ holds.

Equality subgraph: If $l$ is a feasible labeling, let $G_{l}$ denotes a subgraph of $G$; if the condition $l(x)+l(y)=$ $w(x, y)$ is met, then $G_{l}$ is called the equality subgraph with respect to $l$.

Theorem: If $l$ is a feasible labeling of $G$, and $H$ is an optimal matching of $X$ to $Y$ with $H \subseteq G_{l}$, then $H$ is an optimal assignment of $X$ to $Y$. Thus, the problem of finding an optimal assignment is reduced to the problem of finding a feasible vertex labeling of which the equality subgraph contains an optimal matching from $X$ to $Y$.

Applying the K-M algorithm to solve the spectrum handoff subproblem of the interrupted SUs, a weighted bipartite graph $G$ with bipartite division $G^{0}=\left(V_{1}, V_{2}, E\right)$ is constructed, where the set of vertices $V_{1}$ represents the collection of the interrupted SUs, i.e., $\mathrm{V}_{1}=$ $\left[\mathrm{SU}_{1}, \mathrm{SU}_{2}, \ldots, \mathrm{SU}_{M}\right] ; \mathrm{SU}_{m}$ represents the $m$ th interrupted $\mathrm{SU}, 1 \leq m \leq M$; and the set of vertices $V_{2}$ represents the collection of subchannels, i.e., $V_{2}=\left[\mathrm{C}_{1}, \mathrm{C}_{2}, \ldots, \mathrm{C}_{N}\right]$; the weight of the edge $e_{\mathrm{SU}_{m}, \mathrm{C}_{n}}$ is defined as:

$$
w\left(\mathrm{SU}_{m}, \mathrm{C}_{n}\right)=R_{m, n}^{*}-q\left(P_{m, n}^{*}+P^{\mathrm{cir}}\right)
$$


The steps for solving the optimal spectrum handoff subproblem based on the K-M algorithm can be described as follows.

1. Find an initial feasible vertex labeling $l(u)$

2. For given $l(u)$, determine $G_{l}^{0}$ from $G^{0}$ and select a maximum matching $H$.

3. If $H$ is an optimal matching, then the optimization problem formulated in (48) is solved. Otherwise, a label having not being allocated by $H$ is selected in $G_{l}^{0}$. Set $S=V_{1}$, and $T=\Psi$, which denotes the empty set.

4. $N_{G_{l}^{0}}(S)$ denotes the collection of points which connect with $S$ in $G_{l}^{0}$. If $N_{G_{l}^{0}}(S) \neq T$, go to step 3 . Otherwise, $N_{G_{l}^{0}}(S)=T$. Find

$$
\Delta=\min _{u, v}\left(l(u) \mid l(u)+l(v)-w(u, v), u \in S, v \in V_{2}-T\right)
$$

and define

$$
l^{\prime}(u)=\left\{\begin{array}{lc}
l(u)-\Delta, \quad u \in S \\
l(u)+\Delta, \quad u \in T \\
l(u), \quad \text { others. }
\end{array}\right.
$$

5. Replace $l(u)$ by $l^{\prime}(u)$, go back to step 2 .

Through conducting above process iteratively, an optimal matching of $G$ can be obtained corresponding to the optimal spectrum handoff strategy of interrupted SUs.

\section{Simulation results and discussions}

\subsection{Simulation results}

In this section, we examine the performance of the proposed algorithm and compare with two previously proposed algorithms via simulation. In the simulation, we consider a heterogeneous network scenario consisting of one PN and two CRNs, denoted as CRN1 and CRN2, respectively. We assume that all SUs are randomly located in a rectangular region with the size being $100 \mathrm{~m} \times 100 \mathrm{~m}$. The number of PUs in the PN is chosen as 5 , the channel of each PU is divided into 3 or 4 subchannels, and we assume that SUs are allowed to occupy the subchannels of PU1, PU2, and PU3 when accessing CRN1, while are allowed to occupy the subchannels of PU4 and PU5 when accessing CRN2. The subchannel bandwidth of PU1, PU2, and PU3 is chosen as $1 \mathrm{MHz}$ and that of the PU4 and PU5 is chosen as $1.2 \mathrm{MHz}$. The channel model applied in the simulation follows (18), and the noise power is chosen as $-136 \mathrm{dBm}$. The minimum data rate requirement of all the interrupted SUs is set as $1 \mathrm{Mbits} / \mathrm{s}$. The arrival rate and the service rate of the SUs are chosen as 5 and 25 , respectively. To examine the impacts of PUs' service characteristics on the transmission performance of the interrupted SUs, we consider three cases of the combination of $\lambda_{n}^{\mathrm{p}}$ and $\mu_{n}^{\mathrm{p}}$, $1 \leq n \leq N \cdot \lambda_{n}^{\mathrm{p}}$ and $\mu_{n}^{\mathrm{p}}$ and other system parameters used in the simulation are summarized in Tables 1 and 2 .
Table 1 Parameters about the packet arrival rate and service rate of the PUs

\begin{tabular}{lllllll}
\hline PN & PU1 & PU2 & PU3 & PU4 & PU5 \\
\hline Index of subchannel $(n)$ & $1-3$ & $4-6$ & $7-10$ & $11-14$ & $15-18$ \\
Bandwidth of subchannel $(\mathrm{MHz})$ & 1 & 1 & 1 & 1.2 & 1.5 \\
Case 1 & $\lambda_{n}^{(p)}$ & 5 & 10 & 10 & 10 & 5 \\
& $\mu_{n}^{(p)}$ & 15 & 20 & 15 & 20 & 15 \\
Case 2 & $\lambda_{n}^{(p)}$ & 5 & 10 & 10 & 10 & 5 \\
& $\mu_{n}^{(p)}$ & 10 & 20 & 15 & 15 & 10 \\
Case 3 & $\lambda_{n}^{(p)}$ & 8 & 15 & 10 & 12 & 5 \\
& $\mu_{n}^{(p)}$ & 10 & 20 & 15 & 15 & 10 \\
\hline & & & & & &
\end{tabular}

The delay parameters of CRN1 and CRN2 are shown in Table 3. The simulation results are averaged over 1000 independent realizations where each realization involves different realizations of the positions of SUs.

Figure 3 shows the energy efficiency of the interrupted SUs versus the number of iterations obtained from the proposed algorithm. For comparison, we examine the results obtained for different numbers of the interrupted SUs. The maximum transmit power $P_{\max }$ is chosen as $0.1 \mathrm{~W}$, and the service characteristics of PUs are chosen as Case 2 in Table 1. From the figure, we can see that the energy efficiency converges within a small number of iterations for three cases. Comparing the results obtained from different number of SUs, we can see that the energy efficiency of the SUs decreases slightly with the increase of the number of SUs; this is due to the resource competition among the SUs. More specifically, for given subchannel characteristics, in the case that the number of interrupted SUs is small, more flexible and efficient handoff subchannel selection policy might be achieved in terms of the transmission performance of individual SUs, hence resulting in better total energy efficiency performance. However, as the number of interrupted SUs increases, assigning each SU a handoff subchannel with relatively good performance is getting difficult, thus resulting in the degradation in total energy efficiency.

Figure 4 shows the energy efficiency versus the maximum transmit power $\left(P_{m}^{\max }\right)$ of interrupted SUs for different numbers of SUs. To plot the curves, we assume that $P_{m}^{\max }$ is chosen to be the same for all the interrupted SUs. For a fixed $P_{m}^{\max }$, we conduct the proposed scheme and the schemes proposed in $[12,16]$, respectively. In plotting the figure, the service characteristics of PUs is chosen as Case

Table 2 Parameters about maximum tolerable delay of different SUs

\begin{tabular}{lllllllllll}
\hline Index of SU & 1 & 2 & 3 & 4 & 5 & 6 & 7 & 8 & 9 & 10
\end{tabular}

Maximum

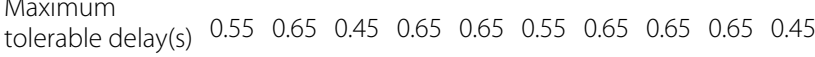


Table 3 Delay parameters

\begin{tabular}{llllllll}
\hline & $t_{\text {prep }}(s)$ & $t_{\text {recfg }}(s)$ & $t_{\text {switch }}(s)$ & $t_{\text {syn }}^{\text {sen }}(s)$ & $t_{\text {sen }}(s)$ & $t_{\text {dec }}(s)$ & $t_{\text {syn }}^{\text {tx }}(s)$ \\
\hline CRN1 & 0.1 & 0.3 & 0.002 & 0.025 & 0.05 & 0.025 & 0.025 \\
CRN2 & 0.1 & 0.3 & 0.002 & 0.025 & 0.025 & 0.025 & 0.025 \\
\hline
\end{tabular}

2 in Table 1 (the same as in Figs. 5 and 6). Based on the obtained handoff subchannel selection and power allocation strategy, the total energy efficiency of the SUs can be examined and is plotted in the figure.

In Fig. 4, comparing the results obtained from the proposed scheme and the schemes proposed in $[12,16]$, we can see that for small $P_{m}^{\max }$, the energy efficiency increases with the increase of $P_{m}^{\max }$ for three algorithms, indicating a larger power threshold is desired for achieving the maximum energy efficiency. However, as $P_{m}^{\max }$ reaches to a certain value, the energy efficiency obtained from our proposed algorithm converges to a fixed value, which will not change with the increase of $P_{m}^{\max }$. The reason is that the optimal energy efficiency has achieved when the transmit power is less than $P_{m}^{\max }$, further increasing $P_{m}^{\max }$ will not change the optimal transmit power; thus, the resulted energy efficiency will not change. On the other hand, when $P_{m}^{\max }$ reaches to a certain value, the energy efficiency obtained from the other two schemes decreases with the increase of $P_{m}^{\max }$. This is because the schemes proposed in $[12,16]$ aim to achieve the maximum data rate and the minimum service time of the SUs, respectively, thus may require higher power consumption, resulting in undesired energy efficiency. Comparing the three curves in the figure, we can see that the proposed scheme outperforms the schemes proposed in $[12,16]$.

Figure 5 shows the energy efficiency versus the maximum transmit power of the interrupted SUs for different circuit power consumption. It can be seen from the figure that the energy efficiency decreases with the increase of circuit power consumption. Comparing the results obtained from the proposed algorithm and the algorithms proposed in $[12,16]$, we can see that the proposed scheme offers higher energy efficiency than the schemes proposed in $[12,16]$.

In Fig. 6, we examine the performance of various spectrum handoff schemes and compare the energy efficiency of the interrupted SUs obtained from the proposed joint optimization scheme and other two schemes, i.e., Schemes 1 and 2. For both Schemes 1 and 2, the optimal power allocation strategy is achieved through maximizing the energy efficiency of each SU-spectrum pair; we then apply different spectrum handoff strategies. For Scheme 1, handoff subchannels are selected in order to achieve the minimum total transmission time of the SUs, while for Scheme 2, we apply random spectrum handoff scheme, i.e., the handoff subchannels of the interrupted SUs are randomly selected. It can be seen from the figure that the proposed algorithm offers better performance in comparison with the other two schemes, indicating that joint design of spectrum handoff and power allocation results in performance enhancement.

In Fig. 7, we examine the energy efficiency obtained from the proposed scheme with different service charac-

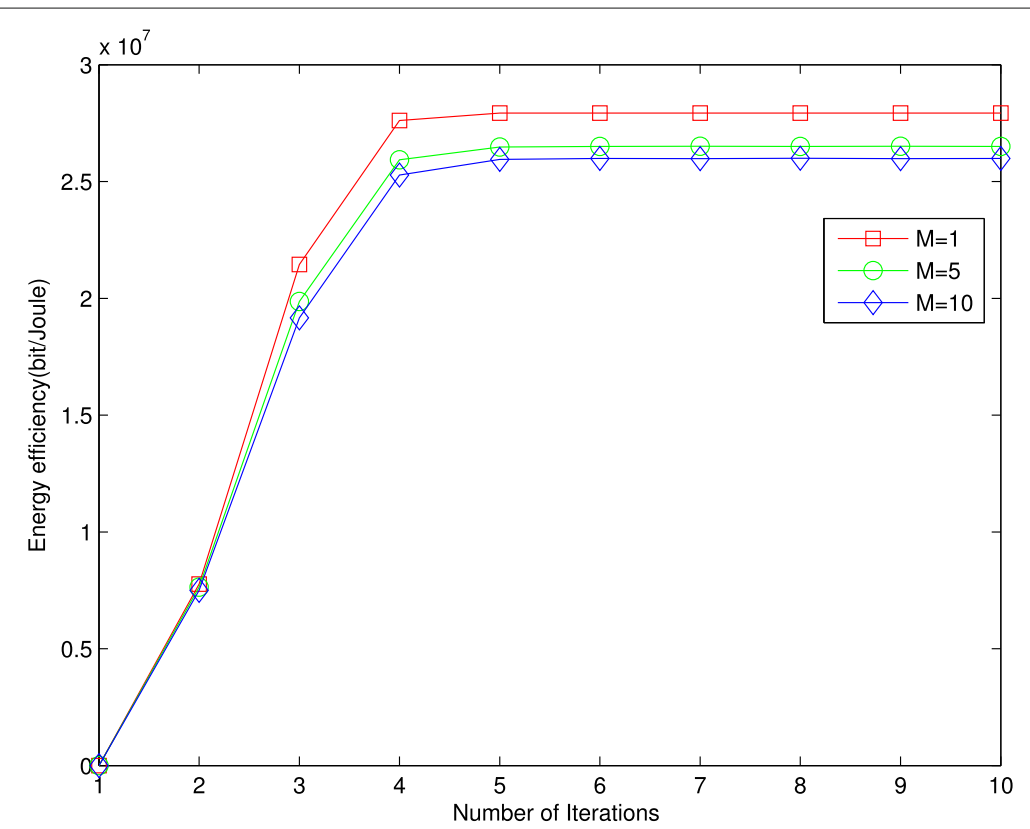

Fig. 3 Energy efficiency versus the number of iterations (different numbers of the interrupted SUs). The energy efficiency of the interrupted SUs versus the number of iterations obtained from the proposed algorithm 


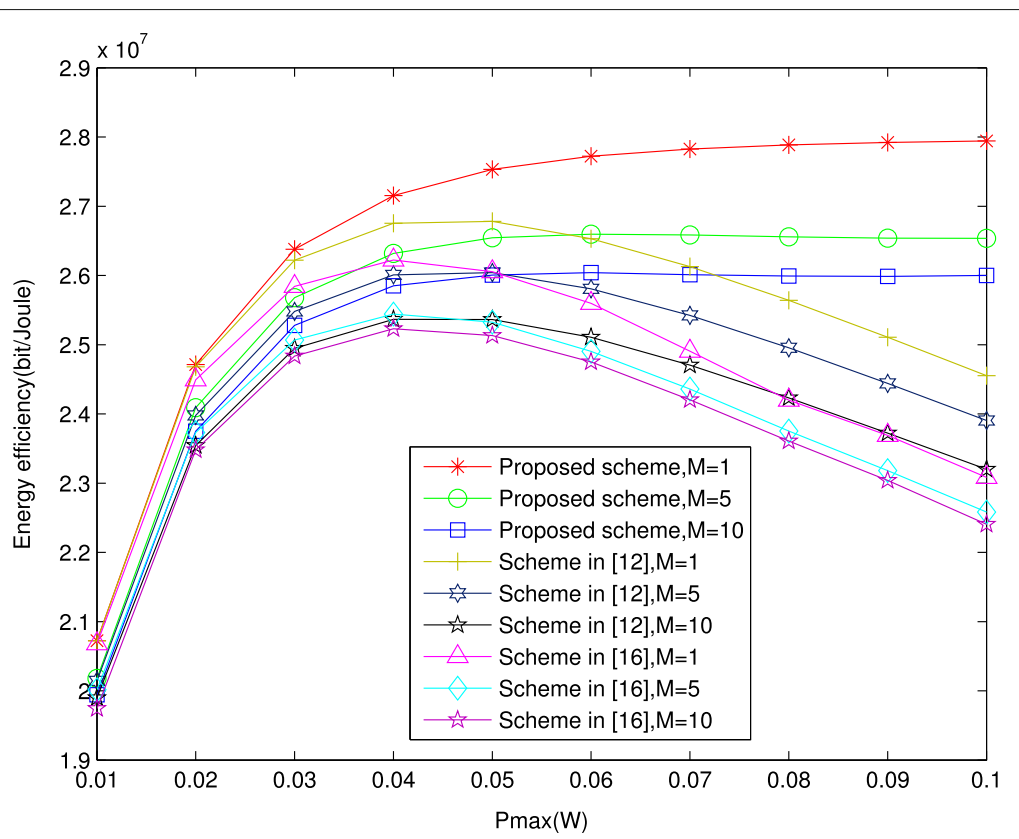

Fig. 4 Energy efficiency versus maximum transmit power (different numbers of the interrupted SUs). The energy efficiency versus the maximum transmit power of the interrupted SUs for different numbers of the SUs

teristics of PUs, including Cases 1, 2, and 3 in Table 1 and that without considering interruption delay constraint. We choose the number of interrupted SUs, i.e., $M$ as 10 in examining the performance. It can be seen from the figure that the scheme without considering interruption delay constraint offers the highest energy efficiency compared to other cases. This is because interruption delay constraint poses requirements on candidate subchannels, thus results in smaller energy efficiency. Comparing the three curves obtained for different service characteristics of PUs, we can see that Case 1 offers the highest energy efficiency whereas Case 3 results in the lowest energy efficiency. The reason is that compared to Cases 2 and 3 , the arrival rate of PUs is relatively small while the service rate is relatively high in Case 1; thus, the average channel busy time resulted from the transmission of the PUs will be small, resulting in more flexible and efficient subchannel selection and better energy efficiency performance in turn.

\subsection{Discussion on channel model}

The channel model considered in this paper is Friis freespace propagation model, where the channel gain of the links between SUs and their corresponding CBSs is constants, determined by the transmitter-receiver separation distance and carrier frequency; thus, for SUs located at different positions, applying different spectrum handoff and power allocation strategies may result in different transmission performance and energy efficiency in turn. In this paper, for given fixed channel characteristics, we jointly consider the transmission performance of a number of handoff SUs and design the optimal spectrum handoff and power allocation strategy for all the handoff SUs.

In wireless communication systems, wireless channel is usually time-varying, which is resulted from the movement of users and the fading effects during signal transmission. However, under the assumption that PUs and SUs move with relatively low speed, we can assume that at interested transmission time, channel characteristics will not change due to user movement. While fading effects may result in the variation of change gain, in the case that the variation of the channel gain is relatively slow, we can also assume that at interested transmission time, channel characteristics are static, i.e., the channel gain is a constant, and design the corresponding spectrum handoff and power allocation scheme.

It should be mentioned that small-scale fading effects caused by multipath propagation may affect link characteristics and transmission performance in turn; however, as small-scale fading effects describe the fluctuations in the received signal envelope relative to the local average of the path loss effects, in the case of week small-scale fading effects, i.e., the average power of the small fading effects, which are commonly modeled as Rayleigh random variables is relatively small, the impacts of small-scale fading on channel characteristics and transmission performance are relatively small compared to pass loss effects, thus can be ignored. In this case, the proposed algorithm in this paper can be applied under acceptable error threshold. 


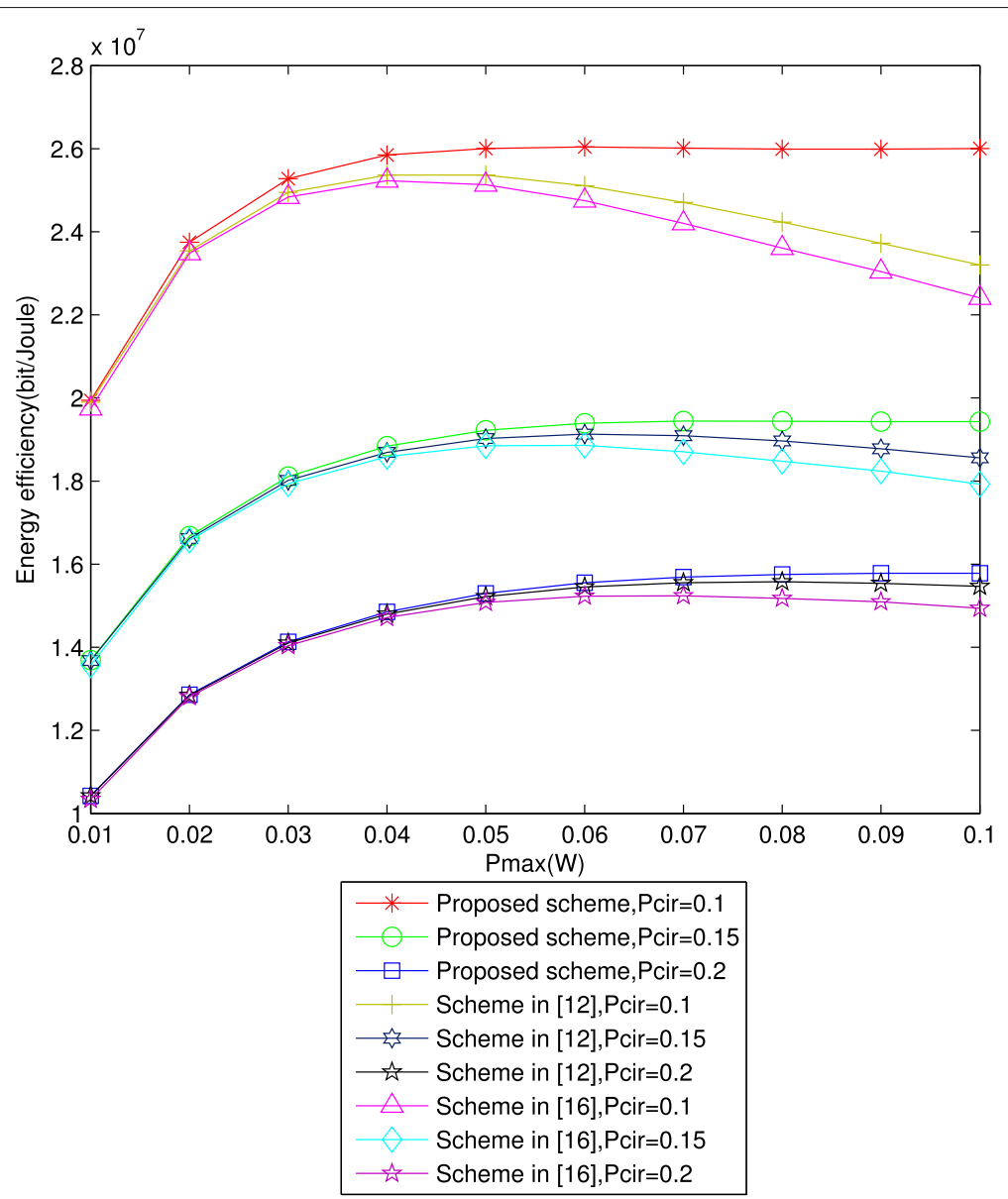

Fig. 5 Energy efficiency versus maximum transmit power (different circuit power). The energy efficiency versus the maximum transmit power of the interrupted SUs for different circuit power consumption

In the case of strong small-scale fading effects, the impacts of channel fading on transmission performance of users may not be ignored and Friis free-space propagation model cannot represent the channel effects accurately. However, as long as the channel characteristics can be obtained in advance, for instance, through applying channel training schemes and the variation of channel characteristics is slow compared to the transmission duration of user information, the basic idea of the proposed algorithm can still be applied to this type of channel scenario. As for given channel characteristics, the energy efficiency of all the SUs can be examined and optimized according to the method proposed in the paper. It should be noticed that in this case, the accuracy of channel estimation may affect the performance of spectrum handoff and power allocation. Although highly accurate channel estimation is desired, it may require large signaling cost and high power consumption; thus, a trade-off between channel estimation and transmission performance should be stressed.
While it is commonly assumed that the channel fading varies relatively slow, thus follows slow fading, it is also possible that the channels between SUs and their corresponding CBSs may experience fast fading, in which case the assumption that the channel characteristics are constants during the transmission periods does not hold, we may not be able to apply the proposed algorithm in a straightforward manner. Instead, we may consider examining the average transmission performance of the channel and the average system performance by taking into account the statistical characteristics of the channels and managing to optimize the average performance of the system. In our future work, we will study the design of spectrum handoff and power allocation algorithms under fading channel scenarios.

\section{Conclusions}

In this paper, an optimal joint spectrum handoff and resource allocation scheme is proposed for interrupted SUs in heterogeneous CRNs. The interruption delay of the SUs is analyzed based on an M/G/1 model, 


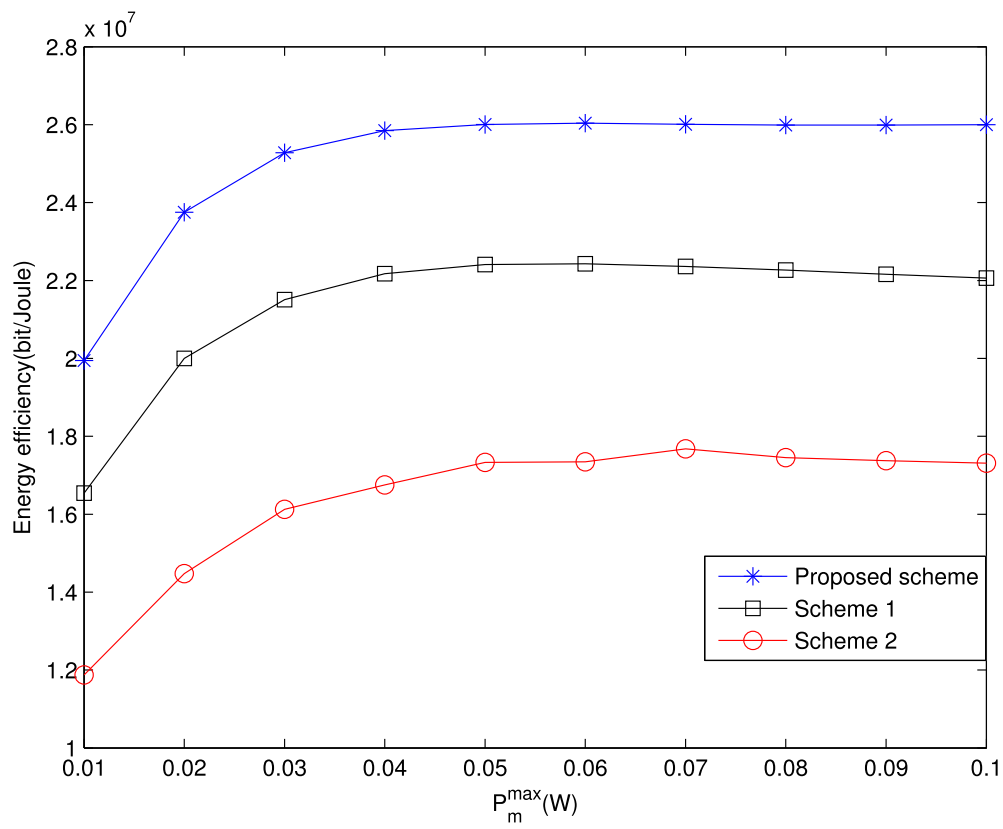

Fig. 6 Proposed joint resource management architecture. The energy efficiency versus the maximum transmit power of the interrupted SUs obtained from the proposed scheme, total transmission time minimization-based spectrum handoff (Scheme 1) and random spectrum handoff (Scheme 2)

and the subchannels which meet the interruption delay and data rate constraints are selected as handoff candidate subchannels. The optimization problem which maximizes the energy efficiency of the interrupted SUs under spectrum handoff, QoS requirement, and the maximum power constraints is formulated and solved through iterative algorithm, Lagrange dual method, and the K-M algorithm. Numerical results demonstrate that compared to the previously proposed algorithms, our proposed energy efficiency-based joint spectrum handoff and resource allocation scheme offers better performance.

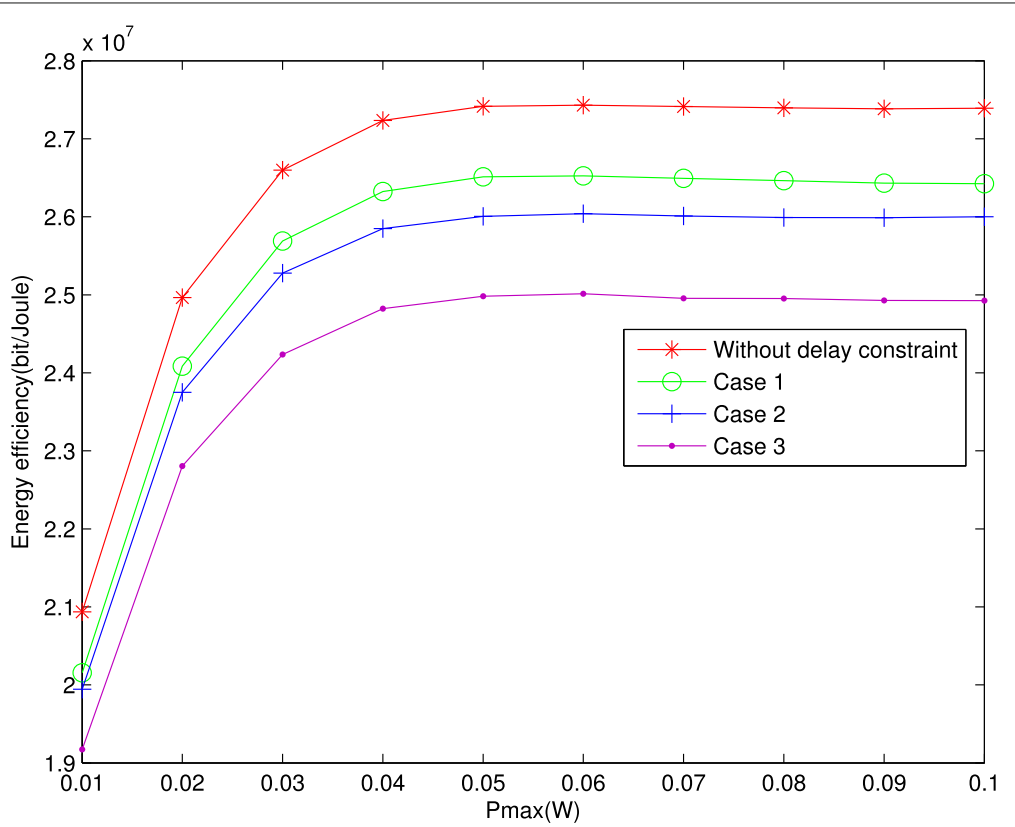

Fig. 7 Energy efficiency versus maximum transmit power (with different service characteristics of PUs and without considering interruption delay constraint). The energy efficiency obtained from the proposed scheme with different service characteristics of PUs and without the interruption delay constraint of the interrupted SUs 


\section{Acknowledgements}

The authors wish to thank the associate editor and the anonymous reviewers for their valuable suggestions, which helped significantly improve the quality of the paper. This work was supported by the 863 project (2014AA01A701), National Science and Technology Specific Project of China (2016ZX03001010-004), the special fund of Chongqing key laboratory (CSTC), and the project of Chongqing Municipal Education Commission (Kjzh11206).

\section{Authors' contributions}

The authors have equal contributions. All authors read and approved the final manuscript.

\section{Competing interests}

The authors declare that they have no competing interests.

Received: 29 January 2016 Accepted: 29 August 2016

Published online: 07 September 2016

\section{References}

1. J Mitola, GQ Maguire, Cognitive radio: making software radios more personal. IEEE Pers. Commun. 6(4), 13-18 (1999). doi:10.1109/98.788210

2. F Sheikholeslami, M Nasiri-Kenari, F Ashtiani, Optimal probabilistic initial and target channel selection for spectrum handoff in cognitive radio networks. IEEE Trans. Wirel. Commun. 14(1), 570-584 (2015). doi:10.1109/TWC.2014.2354407

3. A Mesodiakaki, F Adelantado, L Alonso, C Verikoukis, Performance analysis of a cognitive radio contention-aware channel selection algorithm. IEEE Trans. Veh. Technol. 64(5), 1958-1972 (2015) doi:10.1109/TVT.2014.2341115

4. SWang, F Tosato, J Coon, Reliable energy-efficient spectrum management and optimization in cognitive radio networks: how often should we switch. IEEE Wirel. Commun. 20(6), 14-20 (2013). doi:10.1109/MWC.2013.6704469

5. YQ Wu, FH Hu, S Kumar, YY Zhu, A Talari, N Rahnavard, JD Matyjas, A learning-based qoe-driven spectrum handoff scheme for multimedia transmissions over cognitive radio networks. IEEE J. Sel. Areas Commun. 32(11), 2134-2148 (2014). doi:10.1109/JSAC.2014.141115

6. WY Lee, IF Akyildiz, Spectrum-aware mobility management in cognitive radio cellular networks. IEEE Trans. Mob. Comput. 11(4), 529-542 (2012). doi:10.1109/TMC.2011.69

7. OM Norooz, B Hamdaoui, X Cheng, T Znati, M Guizani, Analyzing cognitive network access efficiency under limited spectrum handoff agility. IEEE Trans. Veh. Technol. 63(3), 1402-1407 (2014). doi:10.1109/TVT.2013.2283856

8. BA Ozan, EB Pehlivanoglu, S Galmes, OB Akan, Dedicated radio utilization for spectrum handoff and efficiency in cognitive radio networks. IEEE Trans. Wirel. Commun. 14(9), 5251-5259 (2015). doi:10.1109/TW.2013.060413.121073

9. MH Yllmaz, MM Abdallah, HM El-Sallabi, J Chamberland, KA Qaraqe, H Arslan, Joint subcarrier and antenna state selection for cognitive heterogeneous networks with reconfigurable antennas. IEEE Trans. Commun. 63(11), 4015-4025 (2015). doi:10.1109/TCOMM.2015.2477088

10. CQ Luo, G Min, FR Yu, Y Zhang, LT Yang, VCM Leung, Joint relay scheduling, channel access, and power allocation for green cognitive radio communications. IEEE J. Sel. Areas Commun. 33(5), 922-932 (2015). doi:10.1109/JSAC.2014.2361085

11. HJ Zhang, CX Jiang, NC Beaulieu, XL Chu, XB Wang, TQS Quek, Resource allocation for cognitive small cell networks: a cooperative bargaining game theoretic approach. IEEE Trans. Wirel. Commun. 14(6), 3481-3493 (2015). doi:10.1109/TWC.2015.2407355

12. R Xie, FR Yu, H Ji, Dynamic resource allocation for heterogeneous services in cognitive radio networks with imperfect channel sensing. IEEE Trans. Veh. Technol. 61(2), 770-780 (2012). doi:10.1109/TVT.2011.2181966

13. CX Jiang, HJ Zhang, Y Ren, HH Chen, Energy-efficient non-cooperative cognitive radio networks: micro, meso, and macro views. IEEE Commun. Mag. 52(7), 14-20 (2014). doi:10.1109/MCOM.2014.6852078

14. C Xiong, L Lu, GY Li, Energy-efficient spectrum access in cognitive radios. IEEE J. Sel. Areas Commun. 32(3), 550-562 (2014) doi:10.1109/JSAC.2014.1403005
15. H Shokri-Ghadikolaei, I Glaropoulos, V Fodor, C Fischione, A Ephremides, Green sensing and access: energy-throughput trade-offs in cognitive networking. IEEE Commun. Mag. 53(11), 199-207 (2015). doi:10.1109/MCOM.2015.7321991

16. LC Wang, CW Wang, CJ Chang, Modeling and analysis for spectrum handoffs in cognitive radio networks. IEEE Trans. Mob. Comput. 11(9), 1499-1513 (2012). doi:10.1109/TMC.2011.155

17. TM Cover, JA Thomas, Elements of information theory. (Wiley, New York, 2012)

18. TS Rappaport, Wireless communications: principles and practice, 2nd edn. (Prentice Hall Communications Engineering and Emerging Technologies Series, New York, 2001)

19. S Boyd, L Vandenberghe, Convex optimization. (Cambridge Univ. Press, Cambridge, 2004)

20. DWK Ng, ES Lo, R Schober, Energy-efficient resource allocation in OFDMA systems with large numbers of base station antennas. IEEE Trans. Wirel. Commun. 11(9), 3292-3304 (2012). doi:10.1109/TWC.2012.072512.111850

21. Q Xu, X Li, H Ji, X Du, Energy-efficient resource allocation for heterogeneous services in OFDMA downlink networks: systematic perspective. IEEE Trans. Veh. Technol. 63(5), 2071-2082 (2014). doi:10.1109/TVT.2014.2312288

22. W Dinkelbach, in Management Science. On nonlinear fractional programming, vol. 13, (1967), pp. 492-498

23. L Lovász, MD Plummer, Matching theory. (American Mathematical Soc, RI, 2009)

\section{Submit your manuscript to a SpringerOpen ${ }^{\circ}$ journal and benefit from:}

- Convenient online submission

- Rigorous peer review

- Immediate publication on acceptance

- Open access: articles freely available online

- High visibility within the field

- Retaining the copyright to your article

Submit your next manuscript at $>$ springeropen.com 